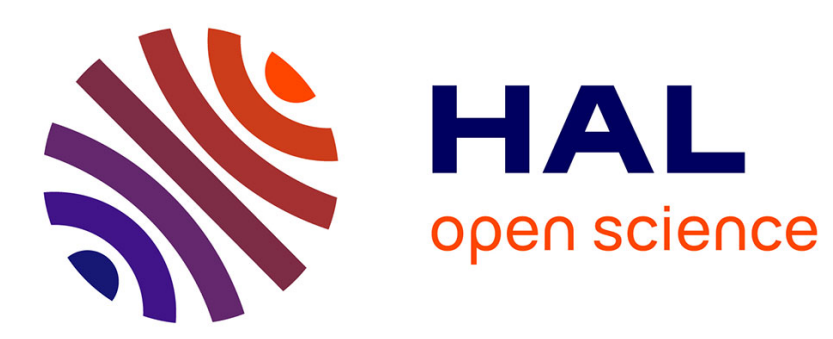

\title{
Activité protéolytique de souches de lactobacilles thermophiles isolées de levains et de Comté. II. Applications en sites industriels
}

\author{
Y Bouton, P Guyot, A Dasen, R Grappin
}

\section{> To cite this version:}

Y Bouton, P Guyot, A Dasen, R Grappin. Activité protéolytique de souches de lactobacilles thermophiles isolées de levains et de Comté. II. Applications en sites industriels. Le Lait, 1994, 74 (1), pp.33-46. hal-00929378

\section{HAL Id: hal-00929378 https://hal.science/hal-00929378}

Submitted on 1 Jan 1994

HAL is a multi-disciplinary open access archive for the deposit and dissemination of scientific research documents, whether they are published or not. The documents may come from teaching and research institutions in France or abroad, or from public or private research centers.
L'archive ouverte pluridisciplinaire HAL, est destinée au dépôt et à la diffusion de documents scientifiques de niveau recherche, publiés ou non, émanant des établissements d'enseignement et de recherche français ou étrangers, des laboratoires publics ou privés. 
(c) Elsevier/INRA

\title{
Activité protéolytique de souches de lactobacilles thermophiles isolées de levains et de Comté. II. Applications en sites industriels
}

\author{
Y Bouton *, P Guyot *, A Dasen, R Grappin
}

Station de recherches en technologie et analyses laitières, INRA, BP 89, 39801 Poligny Cedex, France

(Reçu le 13 avril 1993; accepté le 8 septembre 1993)

\begin{abstract}
Résumé - Cette étude a permis de vérifier en site industriel, l'aptitude protéolytique de souches de lactobacilles thermophiles isolées de levains et de Comté et sélectionnées à partir de tests d'activités sur cultures (dosages des groupements $-\mathrm{NH}_{2}$ par l'acide 2,4,6 trinitrobenzène sulfonique et de l'activité leucine aminopeptidasique). L'importance et la nature de la protéolyse observées sur fromages n'ont pas de relation étroite avec l'activité protéolytique des souches mesurée en laboratoire. En association avec des streptocoques thermophiles, les espèces $L$ delbrueckii subsp lactis produisent davantage d'acides aminés et de petits peptides que les associations avec $L$ helveticus. $L a$ comparaison de profils peptidiques obtenus par CLHP pour les 2 espèces montre que la surface cumulée des pics en fin de chromatogramme, plus importante chez les fromages fabriqués avec les souches de $L$ helveticus $L 79$ et $L 80$, est corrélée avec la note d'amertume.
\end{abstract}

\section{L helveticus / L delbrueckii subsp lactis / fromage / protéolyse / analyse sensorielle}

Summary - Proteolytic activity of thermophilic lactobacilli strains isolated from starters and Comté. II. Applications in cheese plants. The main objectives of this work were to study the influence of lactic bacteria strains on the physicochemical (especially proteolysis) and sensory characteristics of comté. Six Lactobacillus (helveticus and delbrueckii subsp lactis) strains isolated from starters and comté cheeses were chosen according to their proteolytic activity. Regular cheeses were made with different strain associations in 6 local cheese plants. To estimate cheese proteolysis several methods were used: nitrogen fractions by the Kjeldahl method, polyacrylamide-gel electrophoresis of caseins, HPLC of the water soluble fraction, free amino acids by 2,4,6-trinitrobenzenesulfonic acid (TNBS) and leucine aminopeptidase activity (Lap). This work allowed checking in dairies the technological properties of lactic bacteria strains isolated from starters and comté. It was found that the extent and nature of proteolysis in cheese is not correlated with the proteolytic activity measured by laboratory tests. L delbrueckii subsp lactis associations produced more free amino acids and

- Comité interprofessionnel du gruyère de Comté, avenue de la résistance, 39800 Poligny, France. 
small peptides than L helveticus. The comparison of HPLC profiles obtained with the 2 species showed that the cumulative area of peaks at the end of the chromatogram is larger in cheeses made with $L$ helveticus $L 79$ and $L 80$ strains and is correlated with bitterness of the cheeses.

\section{L helveticus / L delbrueckii subsp lactis / cheese / proteolysis / sensory analysis}

\section{INTRODUCTION}

Fromage à pâte cuite pressée, le Comté tout comme le Beaufort est un fromage d'appellation d'origine contrôlée dont la production est limitée à une aire géographique précise. La race et l'alimentation des vaches ainsi que les conditions de collecte du lait et de fabrication sont réglementées. Annuellement, 38000 tonnes de Comté sont produites par près de 250 ateliers de fabrication. Ces fromageries travaillent de façon traditionnelle en utilisant notamment des levains lactiques non sélectionnés dits "sauvages", avec apport occasionnel de levains commerciaux.

La faible teneur en acides aminés (arginine, leucine) dans la fraction non protéique du lait, rend les bactéries lactiques dépendantes de la synthèse d'enzymes protéolytiques : protéinases et peptidases (Khalid et Marth, 1990). Ainsi, seules les bactéries capables de mettre en jeu des systèmes complexes d'enzymes protéolytiques et de transport des acides aminés ou peptides produits pourront croître à leur maximum dans le lait. Le rôle de ces enzymes ne se limite pas à la croissance des bactéries; elles restent en effet actives pendant l'affinage; ainsi les levains, en plus de leur fonction essentielle de production d'acide lactique, participent aux phénomènes de dégradation des protéines du caillé et à la flaveur des fromages. Des études ont montré que la flaveur du Cheddar (Aston et al, 1983) ou celle du Comté (Grappin et Berdagué, 1989) augmente en même temps que la quantité d'acides aminés solubles dans l'acide phosphotungstique. La flaveur du Cheddar n'est d'ailleurs pas reliée à la présence d'un seul acide aminé particulier, mais plutôt à un ensemble d'acides aminés, notamment acide glutamique, glycine, lysine et méthionine (Aston et Dulley, 1982 ; Aston et Creamer, 1986). Dans le Swiss-cheese, les petits peptides et les acides aminés formés contribuent aux goûts de sucré et de noisette ; leur interaction avec le calcium et le magnésium influence le caractère doux et sucré du fromage (Biede et Hammond, 1979).

Après avoir vérifié la capacité technologique en minifabrication d'un petit nombre de souches de bactéries lactiques isolées de levains et de Comté (Bouton et al, 1993), nous avons réalisé des fabrications fromagères en sites industriels. Comme dans l'étude précédente, un des objectifs de ce travail consistait à vérifier les relations existant entre l'activité protéolytique de souches sélectionnées de lactobacilles, mesurée sur lait ou milieu synthétique en laboratoire et le niveau de protéolyse des fromages fabriqués en sites industriels, à partir de ces mêmes souches. Par ailleurs, nous avons essayé de préciser l'influence des facteurs souche et fromagerie (qui comprend les caractéristiques de l'atelier et des techniques de fabrication et d'affinage) sur certaines caractéristiques sensorielles du Comté et de confirmer les liens existant entre la qualité organoleptique et le degré de protéolyse. 


\section{MATÉRIEL ET MÉTHODES}

\section{Souches de bactéries lactiques utilisées}

Les bactéries utilisées dans cette étude proviennent d'une collection de 75 souches destinées à la fabrication de Comté, pour lesquelles nous disposons d'un ensemble d'informations sur leur profil biochimique déterminé à l'aide des galeries API $50 \mathrm{CH}$ et sur leur activité protéolytique et acidifiante. Les souches de lactobacilles ont été choisies en fonction de leur activité protéolytique estimée d'une part, par la mesure des groupements $-\mathrm{NH}_{2}$ par l'acide 2,4,6 trinitrobenzènesulfonique (TNBS) à partir d'une culture sur lait et d'autre part, par la détermination de l'activité leucine-aminopeptidase (Lap) à l'aide du substrat leucine-paranitroanilide. Une ACP réalisée à partir des mesures TNBS et Lap a permis de classer les 50 souches de lactobacilles testées sur la base de leur potentiel protéolytique (Bouton et al, 1993). Afin de se rapprocher de la technologie Comté, nous avons travaillé avec des levains composés d'une part de 2 souches de streptocoques thermophiles S56 et S69, communs à tous les levains et d'autre part, de 2 souches de lactobacilles choisies en fonction de leur activité protéolytique (tableau I). Nous avons ainsi constitué 3 levains composés, outre de streptocoques, de 2 souches de $L$ helveticus $L 72$ et $L 75$ qui présentent de fortes valeurs aux 2 dosages (A1), 2 souches de $L$ helveticus $L 79$ et $\mathrm{L} 80$ qui présentent des valeurs moyennes (A2) et 2 souches de $L$ delbrueckii subsp lactis, $L 85$ qui présente de faibles valeurs et $L 90$ qui a révélé une activité Lap faible, mais de fortes valeurs au dosage des groupements $-\mathrm{NH}_{2}$ (A3). Un quatrième levain (A4), similaire à l'association $\mathrm{A} 3$, comprend en plus, la souche de $S$ salivarius subsp thermophilus $\mathrm{S} 48$ avec laquelle nous avions observé en association, une protéo-

Tableau I. Potentiel protéolytique des souches de $L$ helveticus (L72, L75, L79, L80), de L delbrueckii subsp lactis (L85, L90) et S salivarius subsp thermophilus (S69, S56, S48).

Proteolytic profiles of $L$ helveticus $(L 72, L 75, L 79, L 80)$, $L$ delbrueckii subsp lactis $(L 85, L 90)$ and $S$ salivarius subsp thermophilus (S69, S56, S48) strains.

\begin{tabular}{|c|c|c|c|c|c|c|c|}
\hline \multirow[t]{2}{*}{ Levain } & \multicolumn{3}{|c|}{ Lactobacilles } & \multicolumn{2}{|c|}{ Streptocoques } & \multicolumn{2}{|c|}{ Associations } \\
\hline & Souches & $-\mathrm{NH}_{2}$ & Lap & Souches & Lap & $-\mathrm{NH}_{2}$ & Lap \\
\hline A1 & $\begin{array}{l}\text { L72 } \\
\text { L75 }\end{array}$ & $\begin{array}{l}5,18 \\
4,16\end{array}$ & $\begin{array}{l}37,4 \\
39,5\end{array}$ & $\begin{array}{l}\text { S56 } \\
\text { S69 }\end{array}$ & $\begin{array}{l}13,9 \\
14,7\end{array}$ & 1,65 & 14,5 \\
\hline $\mathrm{A} 2$ & $\begin{array}{l}\text { L79 } \\
\text { L80 }\end{array}$ & $\begin{array}{l}3,17 \\
3,07\end{array}$ & $\begin{array}{l}30,0 \\
29,0\end{array}$ & $\begin{array}{l}\text { S56 } \\
\text { S69 }\end{array}$ & $\begin{array}{l}13,9 \\
14,7\end{array}$ & 1,00 & 13,3 \\
\hline A3 & $\begin{array}{l}\text { L85 } \\
\text { L90 }\end{array}$ & $\begin{array}{l}1,74 \\
4,07\end{array}$ & $\begin{array}{r}9,8 \\
12,7\end{array}$ & $\begin{array}{l}\text { S56 } \\
\text { S69 }\end{array}$ & $\begin{array}{l}13,9 \\
14,7\end{array}$ & 0,78 & 9,7 \\
\hline A4 & $\begin{array}{l}\text { L85 } \\
\text { L90 }\end{array}$ & $\begin{array}{l}1,74 \\
4,07\end{array}$ & $\begin{array}{r}9,8 \\
12,7\end{array}$ & $\begin{array}{l}\text { S56 } \\
\text { S69 } \\
\text { S48 }\end{array}$ & $\begin{array}{l}13,9 \\
14,7 \\
13,7\end{array}$ & 0,54 & 12,3 \\
\hline
\end{tabular}

L'activité leucine-aminopeptidasique (Lap) est exprimée en $\mu \mathrm{g}$ de p-nitroaniline libérés par heure ; les groupements aminés $\left(-\mathrm{NH}_{2}\right)$ sont exprimés en mmol d'équivalent glycine par litre de lait (meq gly/).

Leucine aminopeptidase activity (Lap) is expressed in $\mu \mathrm{g}$ p-nitroaniline/h; free amino acids $\left(-\mathrm{NH}_{2}\right)$ are expressed in mmol equivalent glycine/l milk. 
lyse sur lait plus importante qu'avec la souche S69 (Bouton et al, 1993).

Les mélanges ainsi constitués présentaient tous un $\mathrm{pH}$ voisin de 4,50 après 6 h d'incubation sur lait à $44^{\circ} \mathrm{C}$. Toutes les souches de lactobacilles abaissaient le $\mathrm{pH}$ du lait à $44^{\circ} \mathrm{C}$ jusqu'à 4,6 environ en $8 \mathrm{~h}$ et à une valeur inférieure à 4 en $24 \mathrm{~h}$. Les souches $\mathrm{L} 72$ et $\mathrm{L} 75$ paraissaient les plus sensibles en cycle thermique. La souche de streptocoques S48 était moins acidifiante que les 2 autres dans toutes les conditions (tableau II).

\section{Fabrications fromagères}

Les fabrications ont eu lieu dans 6 ateliers de la région de Franche-Comté avant la période de mise à l'herbe des vaches, pendant 2 mois successifs. La veille de l'expérimentation, les levains ont été préparés au laboratoire sur des milieux utilisés en fromagerie, à partir de cultures mères préparées sur lait écrémé :
- les lactobacilles, ensemencés à $1 \%(v / v)$ sur recuite (lactosérum chauffé à $90^{\circ} \mathrm{C}-95^{\circ} \mathrm{C}$, acidifié à $\mathrm{pH}$ voisin de 5,50 ), sont incubés à $44^{\circ} \mathrm{C}$ pendant $8 \mathrm{~h}$, puis placés à $25^{\circ} \mathrm{C}$ jusqu'au lendemain (pendant environ $8 \mathrm{~h}$ ); le $\mathrm{pH}$ de ces cultures atteint généralement 3,30 ; au moment de l'emploi, la population des mélanges de lactobacilles L72 L75, L79 L80 et L85 L90 atteignait respectivement $2,2,3,8$ et $3,610^{8} \mathrm{UFC} / \mathrm{ml}$;

- les streptocoques thermophiles, ensemencés à $2 \%(\mathrm{v} / \mathrm{v})$ sur sérum thermisé $\left(75^{\circ} \mathrm{C}, 30 \mathrm{~s}\right)$ sont incubés 6 à $7 \mathrm{~h}$ à $44^{\circ} \mathrm{C}$; le $\mathrm{pH}$ atteint généralement 4,50 ; au moment de leur utilisation, la population du mélange de streptocoques S69 S56 atteignait 2,1 $10^{8} \mathrm{UFC} / \mathrm{ml}$, celle de la souche $\mathrm{S} 48,2,910^{8} \mathrm{UFC} / \mathrm{ml}$.

Des levains mésophiles (souche Pal 25, Standa-Industrie, Caen) ont été également préparés par culture après inoculation à $2 \%(\mathrm{v} / \mathrm{v})$ de lactosérum (préalablement porté à $85^{\circ} \mathrm{C}$ pendant 30 s) enrichi à $5 \mathrm{~g} / \mathrm{l}$ de poudre de lait écrémé et incubé $16 \mathrm{~h}$ à $22^{\circ} \mathrm{C}$. Le $\mathrm{pH}$ atteint généralement 4,50 . Au moment de l'utilisation, la population de streptocoques atteignait $1,810^{8} \mathrm{UFC} / \mathrm{ml}$.

Tableau II. Potentiel acidifiant des souches de $L$ helveticus $(L 72, L 75, L 79, L 80)$, de $L$ delbrueckii subsp lactis (L85, L90) et $S$ salivarius subsp thermophilus (S69, S56, S48).

Acidifying profiles of $L$ helveticus $(L 72, L 75, L 79, L 80)$, $L$ delbrueckii subsp lactis $(L 85, L 90)$ and $S$ salivarius subsp thermophilus (S69, S56, S48) strains.

\begin{tabular}{|c|c|c|c|c|c|c|}
\hline \multirow[t]{2}{*}{ Souches } & \multicolumn{2}{|c|}{$p H 6 h$} & \multicolumn{2}{|c|}{$\mathrm{pH} 8 \mathrm{~h}$} & \multicolumn{2}{|c|}{$p H 24 h$} \\
\hline & $44^{\circ} \mathrm{C}$ & Cycle & $44^{\circ} \mathrm{C}$ & Cycle & $44^{\circ} \mathrm{C}$ & Cycle \\
\hline L72 & 5,10 & 6,55 & 4,35 & 6,25 & 3,64 & 5,77 \\
\hline L75 & 5,33 & 6,52 & 4,63 & 6,33 & 3,73 & 5,65 \\
\hline L79 & 5,30 & 6,40 & 4,62 & 5,98 & 3,59 & 3,88 \\
\hline L80 & 5,30 & 6,44 & 4,62 & 6,23 & 3,73 & 4,51 \\
\hline L85 & 5,21 & 6,57 & 4,64 & 6,48 & 3,98 & 4,39 \\
\hline L90 & 5,21 & 6,50 & 4,46 & 6,39 & 3,74 & 3,94 \\
\hline S69 & 4,95 & 6,12 & 4,78 & 5,83 & 4,46 & 4,90 \\
\hline S56 & 5,07 & 6,06 & 4,84 & 5,71 & 4,50 & 4,83 \\
\hline S48 & 5,70 & 6,55 & 5,32 & 6,56 & 4,68 & 5,62 \\
\hline
\end{tabular}

Les valeurs de $\mathrm{pH}$ (exprimées en unité $\mathrm{pH}$ ) sont obtenues après 6,8 et $24 \mathrm{~h}$ d'incubation dans le lait à $44^{\circ} \mathrm{C}$ ou à température variable suivant un cycle programmé reproduisant la succession des températures utilisées en fabrication de Comté.

$\mathrm{pH}$ values (expressed in unity of $\mathrm{pH}$ ) are obtained after 6,8 and $24 \mathrm{~h}$ incubation in milk at $44^{\circ} \mathrm{C}$ or after a thermal cycle that reproduces the temperature evolution of a Comté cheese process. 
L'ensemencement en cuve (addition de $0,15 \%$ de streptocoques mésophiles, $0,15 \%$ de streptocoques thermophiles et $0,10 \%$ de lactobacilles) est réalisé à partir des différentes cultures pures composant les associations. Lorsque la souche $\mathrm{S} 48$ est utilisée, celle-ci est introduite en même temps que les levains mésophiles (addition de 0,05\% de streptocoques \$48 et $0,10 \%$ de streptocoques mésophiles).

Chaque mois, 2 séries de fabrications ont été réalisées dans 6 fromageries $(F 1, F 2, F 3, F 4$, F5, F6). A chaque jour de fabrication, 2 cuves ont été ensemencées avec des associations différentes de souches sélectionnées. Le schéma expérimental correspond à un plan factoriel dont les niveaux sont répartis comme suit : levain (4 niveaux) $x$ fromagerie $(6$ niveaux $) \times$ mois ( 2 répétitions).

\section{Analyses des fromages}

\section{Analyses physico-chimiques}

Des échantillons de fromages ont été prélevés à 3 stades de la fabrication : à $20 \mathrm{~h}, 3$ semaines et 5 mois ; ils ont ensuite été emballés dans du papier d'aluminium sulfurisé puis congelés à $-25^{\circ} \mathrm{C}$ :

- le $\mathrm{pH}$ (exprimé en unité $\mathrm{pH}$ ), est mesuré sur fromage râpé ; une électrode de verre (Radiometer) est introduite dans le fromage tassé dans un godet ; la mesure est relevee lorsque l'équilibre est atteint ;

- l'extrait sec (ES ; exprimé en g pour $100 \mathrm{~g}$ de fromage) est déterminé selon la norme FIL $4 \mathrm{~A}$ (Anonyme, 1982) :

- la matière grasse (MG ; exprimée en g pour $100 \mathrm{~g}$ de fromage) est mesurée par la méthode butyrométrique de Heiss (1961); les dosages d'extrait sec et de matière grasse permettent de définir un autre paramètre : le gras sur $\sec (G)$ S) exprimé en $\mathrm{g}$ pour $100 \mathrm{~g}$ d'extrait sec :

- le chlorure de sodium (exprimé en g pour 100 $g$ d'extrait $\mathrm{sec}$ ) est dosé à l'aide d'un chloruremètre (Corning 926) à électrode soluble d'argent, étalonné par rapport à la technique de dosage des chlorures préconisèe dans la norme FIL 88A (Anonyme, 1988) :

- les isomères $L(+)$ et $D(-)$ du lactate (exprimés en $\mu \mathrm{mol}$ par $\mathrm{g}$ de fromage) sont dosés sur la préparation de l'azote soluble (NS), par la méthode enzymatique Boehringer Mannheim (ref 1112821 , Meylan, France) ;

- azote total et fractions azotées sont déterminés par la méthode Kjeldahl : dosage de l'azote total (NT), de l'azote soluble dans l'acide phosphotungstique (NPT) après préparation selon la technique de Gripon et al (1975) et de l'azote soluble dans l'eau (NS), après préparation selon une technique adaptée de la méthode de Kuchroo et Fox (1982) ; ainsi, $10 \mathrm{~g}$ de fromage additionnés de $50 \mathrm{ml}$ d'eau distillée sont homogénéisés pendant $3 \mathrm{~min}$ à l'aide d'un Ultra-turrax. Cette préparation est placée $30 \mathrm{~min}$ à $40^{\circ} \mathrm{C}$ avant d'être centrifugée à $1200 \mathrm{~g}$ pendant 20 min à $5^{\circ} \mathrm{C}$ Le surnageant est filtré sur filtre Whatman $n^{\circ} 40$; les fractions NS et NPT sont exprimées en pourcentage par rapport à l'azote total (NS/NT et NPT/NT) ; la fraction NPT est également exprimée par rapport à la fraction NS.

- groupements $-\mathrm{NH}_{2}$ dosé par l'acide TNBS ; ce dosage est réalisé sur des dilutions, au $1 / 100^{\circ}$ et $1 / 50^{e}$ respectivement pour les fromages agés de 5 mois et 3 semaines, à partir des préparations d'azote soluble (Bouton et al, 1993) ; les résultats exprimés en équivalent glycine sont convertis en pourcentage par rapport à l'azote total $\left(-\mathrm{NH}_{2} / \mathrm{NT}\right)$;

- chromatographie liquide haute performance en phase inverse sur les fromages affinés (RPCLHP) : cette technique est utilisée pour évaluer les peptides contenus dans les fromages; les analyses sont effectuées sur la fraction NS; les échantillons sont filtrés sur filtres Millipore 0,45 $\mu \mathrm{m}$; le filtrat, contenant les acides aminés et les peptides est injecté sur colonne CLHP thermostatée à $30^{\circ} \mathrm{C}$, à l'aide d'un bain-marie ; la chromatographie en "phase inverse" est réalisée sur un appareil Varian 5000, muni d'un passeur automatique d'échantillons Kontron 465, d'un détecteur UV et d'un intégrateur Spectraphysics SP4290, permettant l'intégration de chaque pic. Les conditions chromatographiques sont les suivantes : phase : silice poreuse (diamètre $5 \mu \mathrm{m}$, porosité $300 \AA$ ) greffée $\mathrm{C} 18$ (endcapped) ; précolonne : $15 \mathrm{~mm} \times 4,6 \mathrm{~mm}$ : colonne: $225 \mathrm{~mm} \times 4,6 \mathrm{~mm}$; temperature colonne: $30^{\circ} \mathrm{C}$; débit : $0,8 \mathrm{ml} / \mathrm{min}$; longueur d'onde : $214 \mathrm{~nm}$; sensibilité détection : 0,002 Unité Arbitraire/mV ; atténuation enregistreur : 128 ; volume injecté : $20 \mu \mathrm{l}$.

Les échantillons sont élués par un gradient de solvants : les solvants purs sont filtrés sur filtre de porosité $0,45 \mu \mathrm{m}$ et dégazés aux ultra- 
sons, sous vide ; le solvant aqueux (ou solvant A) est représenté par un mélange d'eau ultrapure (résistivité $18 \mathrm{~m} \Omega$ ) avec $0,1 \%$ d'acide trifluoroacétique (TFA) ; le solvant organique (ou solvant $\mathrm{B}$ ) est constitué d'un mélange d'acétonitrile $\left(\mathrm{CH}_{3} \mathrm{CN}\right)$ et d'eau ultra-pure $(60 / 40, \mathrm{v} / \mathrm{v})$ additionné de $0,1 \%$ de TFA.

Pour l'exploitation des résultats, les profils chromatographiques ont été divisés en 4 zones comprises entre 4 pics majeurs présents sur tous les chromatogrammes.

\section{Électrophorèses des caséines}

Les principales fractions caséines et leurs produits de dégradation $\left(\alpha_{s 1}, \alpha_{s 1-1}, \beta\right.$, " $\beta$-dégradée", $\left.\gamma_{1}, \gamma_{2}, \gamma_{3}\right)$ sont suivis par électrophorèse sur gel de polyacrylamide-agarose (PAGE), d'après la technique d'Uriel (1966), modifiée par Collin et al (1987). L'intensité de coloration des bandes d'électrophorèse est suivie à l'aide d'un densitomètre Vernon pHI3 (longueur d'onde $400 \mathrm{~nm}$ ). Chaque fraction caséine est exprimée en pourcentage de l'aire totale des fractions étudiées.

\section{Activité leucine-aminopeptidasique}

Elle est exprimée en $\mu g$ de p-nitro-aniline libéré par $\mathrm{h}$ et par $\mathrm{g}$ de fromage et décrite dans un article précédent (Bouton et al, 1993).

\section{Acides gras volatils (AGV)}

Exprimés en $\mathrm{mg}$ pour $100 \mathrm{~g}$ de fromage, ils sont dosés par chromatographie en phase gazeuse après extraction (Berdagué et al, 1987). L'appareil utilisé est un chromatographe de type IGC $121 \mathrm{DFL}$ (Intersmat) relié à un intégrateurcalculateur ICR 1B (Intersmat).

Les conditions chromatographiques sont les suivantes : colonne : inox, $2 \mathrm{~m} \times 2,5 \mathrm{~mm}$, silice Chromosorb G AW DMCS, granulométrie 100120 mesh, enrobé à 10\% par FFAP (free fatty acid phase); gaz (qualité C) : $P(N 2)=1,1$ bar, $P(H 2)=0,9$ bar, $P($ air $)=1,3$ bar ; températures: injecteur et détecteur : $210^{\circ} \mathrm{C}$, four : gradient, $145^{\circ} \mathrm{C}(10 \mathrm{~min})$ puis augmentation de $6^{\circ} \mathrm{C} / \mathrm{min}$ jusqu'à $180^{\circ} \mathrm{C}(8 \mathrm{~min})$; sensibilité : $10^{-11} \mathrm{~A} / \mathrm{mV}$; volume d'échantillon : $0,5 \mu \mathrm{l}$.

\section{Analyses sensorielles}

Les analyses sensorielles ont porté sur l'ensemble des 48 fromages fabriqués. Les 2 fro- mages provenant d'une même fromagerie et d'un même jour de fabrication ont été analysés ensemble, au stade coupe (5 mois).

Les évaluations sensorielles étaient de type analytique descriptive, utilisant une échelle de notation à 6 barreaux (intervalles structurés) en vue d'analyses qualitatives et quantitatives, les notes pouvant s'échelonner de 0 à 10 . Le jury était formé d'un groupe de 14 personnes entraînées. Les fromages ont été notés sur des critères de goût et de pâte (Lavanchy et al, 1993).

\section{Critères utilisés pour la caractérisation du goût}

Ces critères sont les suivants :

- intensité : indépendamment de sa finesse ;

- typicité : finesse du goût ;

- amertume ;

- acidité ;

- piquant : sensation perçue sur la langue pendant la mastication.

\section{Critères utilisés pour la caractérisation de la pâte}

Il s'agit de :

- résistance : résistance opposée à une compression importante (consistance molle à dure):

- onctuosité : impression de matière grasse ;

- élasticité : appréciation du retour à l'état initial, sans fissure, après une contrainte modérée $(30 \%)$;

- hétérogénéité de la structure : perception d'une macrostructure en cours de déglutition.

Au vu de ces résultats, chaque dégustateur a attribué pour chaque fromage une note globale de goût, et de pâte. Les dégustations se sont déroulées dans un local climatisé à $18^{\circ} \mathrm{C}$, les boxes de dégustations individuels étant éclairés en lumière jaune, afin d'éliminer les interactions éventuelles entre la couleur des fromages et les caractéristiques sensorielles. Les fromages, dècoupés en petits cubes de $1 \mathrm{~cm}$ d'arête, étaient présentés dans des boîtes de Pétri de $8 \mathrm{~cm}$ de diamètre ; les dégustateurs étaient invités à se rincer la bouche (eau de Volvic) entre chaque dégustation. Des tranches de pain étaient mises à leur disposition pendant la séance. Pour chaque caractère sensoriel, une note correspondant à la moyenne des notes du jury, a été attribuée à chaque fromage. Chaque moyenne a 
été intégrée dans l'analyse statistique au même titre qu'une valeur analytique.

\section{Traitements des résultats}

Bien que le nombre de répétitions soit toujours faible, une exploitation statistique a néanmoins été réalisée. Les analyses ont été effectuées à partir du logiciel Stat-ITCF (Version 4, 1987) de I'Institut technique des céréales et des fourrages. Une analyse de variance a permis d'étudier les facteurs souche et fromagerie sur la composition et la qualité finale des fromages; lorsque l'un des facteurs s'est révélé significatif, le test de Newman-Keuls, basé sur la plus petite différence existant entre les différentes moyennes, a été appliqué afin de classer les fromages.

\section{RÉSULTATS ET DISCUSSION}

\section{Caractéristiques physico-chimiques}

\section{Composition des fromages (tableau III)}

Les variations de $\mathrm{pH}(+0,55)$, d'extrait sec $(+3,7 \mathrm{~g} / 100 \mathrm{~g})$ et de lactates $(-41 \%)$ en cours d'affinage sont en accord avec les observations faites par Grappin et Berdagué (1989) sur Comté.

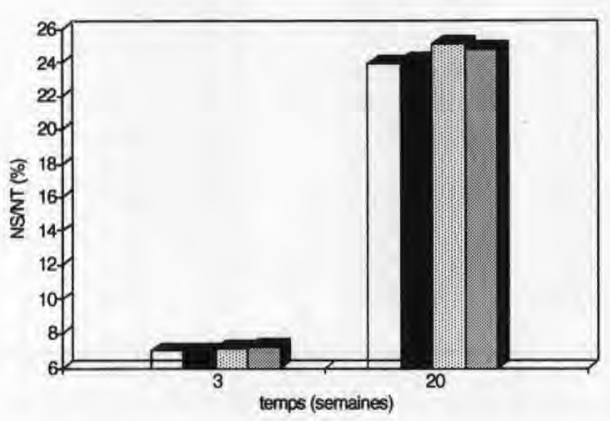

Les teneurs en acides gras volatils obtenues sur les fromages de 5 mois (résultats non montrés) sont similaires à celles obtenues par Berdagué et al, 1987 (C2 = 200, $\mathrm{C} 4=17, \mathrm{iC} 5=6$ et $\mathrm{C} 6=5 \mathrm{mg} / 100 \mathrm{~g}$ ). À ce stade de l'affinage, l'acide acétique est très corrélé à l'acide propionique $(r=0,906)$; ces acides ( $\mathrm{C} 2$ et $\mathrm{C} 3$ ) sont inversement corrélés aux teneurs en lactates. Ces résultats indiquent que les fromages ont eu un affinage normal.

Les rapports NS/NT, NPT/NT et $-\mathrm{NH}_{2} /$ NT augmentent au cours de l'affinage, respectivement de $7,0 \%$ à $24,5 \%$, de $2,0 \%$ à $10,1 \%$ et $1,5 \%$ à $7,3 \%$. Les associations de $L$ helveticus $A 1$ et $A 2$ présentent une cinétique de protéolyse similaire (fig 1a, 1b) de même que les associations de $L$ delbrueckii subsp lactis A3 et A4. Si la différence de hauteur est peu marquée pour les 2 espèces quant au rapport NS/NT, elle l'est davantage pour le rapport NPT/NT. Ainsi, les fromages fabriqués avec les associations de souches $A 3$ et $A 4$ sont protéolysés plus vite que ceux issus des associations $A 1$ et $A 2$.

Les fractions caséines évaluées au stade coupe sont similaires à celles observées par Collin et al, $1987(\beta=18,71$, $\beta$ deg $=2,64, \gamma_{1}=9,49, \gamma_{2}=6,54, \gamma_{3}=7,41$, $\alpha_{s 1}=13,78, \alpha_{s 1-1}=28,68 \%$ ).

b

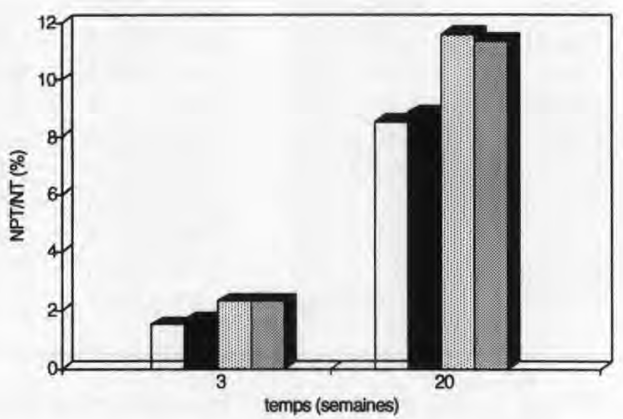

Fig 1. Évolution des fractions azotées NS/NT $A 2, \cong A 3, \because A 4$.

(a) et NPT/NT (b) au cours de l'affinage. A1, Evolution of water soluble nitrogen in $100 \mathrm{~g}$ of total $\mathrm{N}$ (a) and phosphotungstic acid soluble nitrogen in $100 \mathrm{~g}$ of total $\mathrm{N}(\mathrm{b})$ fraction during ripening. 
Tableau III. Analyses physico-chimiques et sensorielles des fromages de 5 mois (moyenne \pm écarttype),

Physico-chimical and sensory measurements on $4 \times 12$ five-month-old cheeses (mean \pm standard deviation).

\begin{tabular}{|c|c|c|c|c|c|}
\hline & $A 1$ & $A 2$ & $A 3$ & $A 4$ & Moyenne \\
\hline $\mathrm{pH}$ & $5,74 \pm 0,05$ & $5,75 \pm 0,09$ & $5,83 \pm 0,07$ & $5,83 \pm 0,12$ & $5,79 \pm 0,10$ \\
\hline ES & $64,03 \pm 1,01$ & $63,80 \pm 0,85$ & $63,98 \pm 0,63$ & $63,73 \pm 0,79$ & $63,89 \pm 0,84$ \\
\hline $\mathrm{G} / \mathrm{S}$ & $48,38 \pm 1,54$ & $49,30 \pm 1,36$ & $49,60 \pm 1,34$ & $49,79 \pm 1,32$ & $49,52 \pm 1,41$ \\
\hline $\mathrm{NaCl}$ & $1,39 \pm 0,36$ & $1,52 \pm 0,20$ & $1,30 \pm 0,39$ & $1,42 \pm 0,25$ & $1,41 \pm 0,32$ \\
\hline Lap $20 \mathrm{~h}$ & $143,4 \pm 54,4$ & $170,7 \pm 50,5$ & $127,1 \pm 29,6$ & $116,0 \pm 35,6$ & $139,3 \pm 48,3$ \\
\hline NS/NT & $23,95 \pm 1,30$ & $24,14 \pm 1,62$ & $25,05 \pm 1,77$ & $24,80 \pm 2,06$ & $24,49 \pm 1,77$ \\
\hline NPT/NS & $35,69 \pm 5,21$ & $36,57 \pm 5,07$ & $46,18 \pm 3,76$ & $45,49 \pm 6,07$ & $40,98 \pm 7,05$ \\
\hline$-\mathrm{NH}_{2} / \mathrm{NT}$ & $6,49 \pm 0,78$ & $6,56 \pm 0,73$ & $7,95 \pm 0,68$ & $8,01 \pm 1,37$ & $7,25 \pm 1,18$ \\
\hline NPT/NT & $8,53 \pm 1,19$ & $8,85 \pm 1,48$ & $11,57 \pm 1,47$ & $11,36 \pm 2,29$ & $10,08 \pm 2,17$ \\
\hline$\beta$ & $18,67 \pm 2,85$ & $19,29 \pm 2,55$ & $18,13 \pm 1,90$ & $18,18 \pm 2,03$ & $19,03 \pm 2,35$ \\
\hline$\beta$ deg & $2,64 \pm 0,55$ & $2,77 \pm 0,34$ & $3,08 \pm 0,93$ & $2,95 \pm 0,65$ & $2,86 \pm 0,63$ \\
\hline$\gamma_{1}$ & $9,81 \pm 0,53$ & $9,86 \pm 0,65$ & $10,31 \pm 1,65$ & $10,23 \pm 0,60$ & $9,95 \pm 1,02$ \\
\hline$\gamma_{2}$ & $7,10 \pm 1,06$ & $7,19 \pm 1,01$ & $7,6 \pm 0,89$ & $7,32 \pm 1,16$ & $7,27 \pm 0,99$ \\
\hline$\gamma_{3}$ & $8,26 \pm 0,85$ & $8,14 \pm 0,98$ & $8,23 \pm 0,74$ & $8,18 \pm 0,86$ & $8,16 \pm 0,86$ \\
\hline$\alpha_{s 1}$ & $15,08 \pm 1,58$ & $14,97 \pm 2,10$ & $14,52 \pm 2,33$ & $14,38 \pm 1,96$ & $14,60 \pm 2,18$ \\
\hline$\alpha_{s 1-1}$ & $24,50 \pm 2,42$ & $24,32 \pm 2,01$ & $25,20 \pm 1,65$ & $25,48 \pm 2,76$ & $24,95 \pm 2,42$ \\
\hline Pic 1 & $10,98 \pm 3,57$ & $9,71 \pm 3,31$ & $13,31 \pm 3,31$ & $14,29 \pm 4,80$ & $12,07 \pm 4,21$ \\
\hline Zone 1 & $11,11 \pm 2,15$ & $10,28 \pm 2,52$ & $11,89 \pm 2,52$ & $10,76 \pm 3,47$ & $11,01 \pm 2,95$ \\
\hline Pic 2 & $8,11 \pm 3,37$ & $7,29 \pm 1,62$ & $10,23 \pm 1,62$ & $9,17 \pm 2,04$ & $8,70 \pm 2,56$ \\
\hline Zone 2 & $1,37 \pm 0,19$ & $1,84 \pm 0,58$ & $1,61 \pm 0,58$ & $1,66 \pm 0,52$ & $1,62 \pm 0,51$ \\
\hline Pic 3 & $14,07 \pm 3,79$ & $13,51 \pm 3,10$ & $15,90 \pm 3,10$ & $17,61 \pm 4,03$ & $15,27 \pm 3,81$ \\
\hline Zone 3 & $5,71 \pm 1,43$ & $6,04 \pm 1,47$ & $4,57 \pm 1,47$ & $4,42 \pm 1,24$ & $5,18 \pm 1,57$ \\
\hline Pic 4 & $8,73 \pm 2,70$ & $7,83 \pm 4,98$ & $10,94 \pm 4,98$ & $11,40 \pm 7,16$ & $9,73 \pm 5,48$ \\
\hline Zone 4 & $39,91 \pm 11,71$ & $43,40 \pm 9,53$ & $31,15 \pm 9,53$ & $31,09 \pm 8,72$ & $36,39 \pm 11,41$ \\
\hline Pâte & $6,68 \pm 0,36$ & $6,49 \pm 0,46$ & $6,44 \pm 0,51$ & $6,39 \pm 0,70$ & $6,50 \pm 0,53$ \\
\hline Goût & $5,86 \pm 0,61$ & $5,94 \pm 0,46$ & $6,04 \pm 0,43$ & $6,25 \pm 0,49$ & $6,02 \pm 0,52$ \\
\hline Résist & $5,06 \pm 0,77$ & $5,32 \pm 0,83$ & $5,67 \pm 0,86$ & $5,65 \pm 1,24$ & $5,42 \pm 0,97$ \\
\hline Onct & $5,96 \pm 0,80$ & $5,83 \pm 0,68$ & $5,47 \pm 0,77$ & $5,68 \pm 1,20$ & $5,73 \pm 0,92$ \\
\hline Elast & $5,52 \pm 0,74$ & $5,49 \pm 1,17$ & $5,71 \pm 0,81$ & $5,69 \pm 0,85$ & $5,60 \pm 0,91$ \\
\hline Hétér & $3,46 \pm 0,72$ & $3,62 \pm 0,78$ & $3,88 \pm 0,71$ & $4,02 \pm 1,15$ & $3,74 \pm 0,89$ \\
\hline Intens & $5,82 \pm 0,48$ & $6,03 \pm 0,42$ & $6,05 \pm 0,45$ & $6,15 \pm 0,57$ & $6,01 \pm 0,50$ \\
\hline Typicité & $4,52 \pm 0,63$ & $4,73 \pm 0,56$ & $4,94 \pm 0,48$ & $4,93 \pm 0,65$ & $4,78 \pm 0,61$ \\
\hline Amer & $3,95 \pm 1,50$ & $3,86 \pm 0,77$ & $2,69 \pm 0,73$ & $2,71 \pm 0,54$ & $3,30 \pm 1,13$ \\
\hline Acidité & $2,21 \pm 0,37$ & $2,12 \pm 0,36$ & $2,41 \pm 0,40$ & $2,22 \pm 0,30$ & $2,24 \pm 0,37$ \\
\hline Piquant & $1,75 \pm 0,53$ & $1,97 \pm 0,67$ & $2,17 \pm 0,56$ & $2,15 \pm 0,53$ & $2,01 \pm 0,60$ \\
\hline
\end{tabular}

$A 1, A 2, A 3, A 4$ : moyenne des 12 fromages essais fabriqués respectivement avec les associations $A 1, A 2, A 3, A 4$; ES : extrait sec ( $\mathrm{g} / 100 \mathrm{~g}$ fromage) ; G/S : gras sur sec; $\mathrm{NaCl}$ ( $\mathrm{g} / 100 \mathrm{~g} \mathrm{ES}$ ) ; Lap : activité leucine-aminopeptidasique ( $\mu \mathrm{g} \mathrm{PNa/h/g);} \mathrm{fractions} \mathrm{azotées} \mathrm{(} g / 100 \mathrm{~g} \mathrm{NT}$ ) ; caséines et surface des pics (fig 2), (\% par rapport à la surlace totale) ; Résist : résistance, Onct : onctuosité, Elast : élasticité, Hétér : hétérogénéité, Intens : intensité, Amer : amertume (note /10).

Average results obtained with 12 cheeses made with A1, A2, A3, A4 associations; ES: dry matter ( $g / 100 \mathrm{~g}$ cheese); $\mathrm{G} / \mathrm{S}$ fat on dry matter; $\mathrm{NaCl}$ ( $g / 100 \mathrm{~g}$ ES); Lap: leucine-aminopeptidase activity ( $\mu \mathrm{g}$ PNa/h/g); nitrogen fractions ( $g$ / $100 \mathrm{~g}$ of total nitrogen); caseins, "pic" and "zone" (fig 2), (\% of total surface); résist: hardness, onct: unctuousness, elast: springiness, hétér: heterogeneity, intens: taste intensity, amer: bitterness (score/10). 


\section{Influence des facteurs}

L'influence des facteurs souche et fromagerie a été évaluée à l'aide d'une analyse de variance des résultats au stade coupe (tableau IV).

Le facteur souche est hautement significatif pour les variables $-\mathrm{NH}_{2} / \mathrm{NT}$ et NPT/ NT expliquant respectivement $38 \%$ et $41 \%$ des variations. D'après le classement de Newman-Keuls, les souches sont regroupées selon leur espèce ; ainsi, les associations de $L$ delbrueckii subsp lactis constituent un groupe homogène donnant des fromages plus finement protéolysés (rapports NPT/NT et $-\mathrm{NH}_{2} / \mathrm{NT}$ élevés) que ceux issus des fromages fabriqués avec les associations de $L$ helveticus.

Le facteur fromagerie explique $19 \%$ et $34 \%$ des variations observées pour le rapport NPT/NS et le taux de caséines $\gamma_{2}$. Collin et al (1987) avaient également observé l'importance du facteur fromagerie (composition du lait, technologie) sur la fraction NS et les différentes caséines. Les fromages provenant de la fromagerie $\mathrm{F} 4$ ont un taux de petits peptides et d'acides aminés et une proportion de $\gamma_{2}$ plus importants que ceux des autres fromageries. Aucune corrélation significative n'a pu être mise en évidence entre les teneurs en plasmine et plasminogène des laits utilisés en fromagerie et celle des caséines $\gamma$ et $\beta$ deg ; toutefois, les résultats plus élevés de la fromagerie F4 peuvent s'expliquer par une température d'affinage plus haute (Delacroix-Buchet et Fournier, 1992).

\section{Comparaison de la protéolyse observée sur les cultures et sur les fromages}

Le coefficient de corrélation $(0,499)$ obtenu entre les valeurs d'activité Lap mesurées sur les cultures et celles observées sur les fromages n'est pas significatif. Si l'association A1 donne l'activité Lap la plus élevée en laboratoire (tableau I), c'est l'association A2 qui, sur fromage à $20 \mathrm{~h}$, donne l'activité la plus élevée (tableau III). Toutefois, les ac-
Tableau IV. Analyse de variance des mesures physico-chimiques réalisées sur fromages de 5 mois.

Analysis of variance of the physico-chemical measurements made on 48 five-month-old cheeses.

\begin{tabular}{|c|c|c|c|c|}
\hline \multirow[t]{2}{*}{ Analyses } & \multicolumn{2}{|c|}{$\begin{array}{l}\text { Facteur } \\
\text { souche }\end{array}$} & \multicolumn{2}{|c|}{$\begin{array}{l}\text { Facteur } \\
\text { fromagerie }\end{array}$} \\
\hline & 1 & 2 & 1 & 2 \\
\hline $\mathrm{pH}$ & 1 & & I & \\
\hline ES & NS & & NS & \\
\hline G/S & NS & & $\cdot$ & 36 \\
\hline $\mathrm{NaCl}$ & NS & & 1 & \\
\hline Lap $20 \mathrm{~h}$ & 1 & & 1 & \\
\hline NS/NT & NS & & 1 & \\
\hline NPT/NS & 1 & & * & 19 \\
\hline$-\mathrm{NH}_{2} / \mathrm{NT}$ & $\star \star * *$ & 38 & * & 17 \\
\hline NPT/NT & $* * * *$ & 41 & $* *$ & 23 \\
\hline$\beta$ & 1 & & 1 & \\
\hline Bdeg & 1 & & 1 & \\
\hline$\gamma_{1}$ & 1 & & 1 & \\
\hline$\gamma_{2}$ & 1 & & "* & 34 \\
\hline$\gamma_{3}$ & NS & & 1 & \\
\hline$\alpha_{s 1}$ & NS & & 1 & \\
\hline$\alpha_{s 1-1}$ & NS & & 1 & \\
\hline Pic 1 & . & 19 & - & 22 \\
\hline Zone 1 & NS & & NS & \\
\hline Pic 2 & 1 & & 1 & \\
\hline Zone 2 & 1 & & NS & \\
\hline Pic 3 & NS & & & \\
\hline Zone 3 & * & 20 & NS & \\
\hline Pic 4 & 1 & & 1 & \\
\hline Zone 4 & * & 22 & NS & \\
\hline Pâte & NS & & NS & \\
\hline Goût & NS & & NS & \\
\hline Résistance & 1 & & $\cdots$ & 40 \\
\hline Onctuosité & NS & & $*$ & 37 \\
\hline Élasticité & NS & & $* * *$ & 60 \\
\hline Hétérogène & NS & & . & 28 \\
\hline Intensité & NS & & NS & \\
\hline Typicité & NS & & NS & \\
\hline Amertume & 1 & & NS & \\
\hline Acidité & NS & & $*$ & 32 \\
\hline Piquant & NS & & 1 & \\
\hline
\end{tabular}

1 : Seuil de signification : non significatif (NS), significatif au seuil de $5 \%\left({ }^{*}\right)$, de $1 \%\left({ }^{* *}\right)$, de $0,1 \%\left({ }^{* *}\right) ; 2$ : pourcentage de la variance expliquée par le facteur ; (/) aucune conclusion n'a pu être donnée, les variances entre traitements n'étant pas homogènes.

1: significant level : non significant (NS); significant $\mathrm{P}<$ $0.05\left(^{*}\right)$, significant $\mathrm{P}<0.01(*)$, significant $\mathrm{P}<0.001$ $(* *) ; 2$ : percent of the variance explained by the factor; (/) the variance between treatments being not homogeneous, no conclusion can be drawn. 
tivités mesurées sur les fromages issus des souches de $L$ helveticus restent supérieures, comme en tests de laboratoire, à celles développées par les souches de $L$ delbrueckii subsp lactis. Ce résultat indique que les souches de lactobacilles se sont bien développées pendant la fabrication.

Par ailleurs, il existe une corrélation négative $(-0,923)$ entre les mesures des groupements aminés effectuées au laboratoire et celles réalisées sur les fromages de 5 mois. Ainsi, l'association A1 qui présentait en laboratoire le taux de groupements aminès le plus élevé, de mëme qu'une activité Lap élevée donne à 5 mois les fromages les moins protéolysés.

Ces informations contradictoires obtenues au laboratoire et sur fromages peuvent peut-être s'expliquer par une légère différence de $\mathrm{pH}$ entre les fromages. En effet, même si le dispositif expérimental ne permet pas de conclure à des différences significatives, les fromages issus des associations $\mathrm{A} 1$ et $\mathrm{A} 2$ présentent des valeurs de $\mathrm{pH}$ à 5 mois légèrement plus faibles que ceux issus des associations $\mathrm{A} 3$ et $\mathrm{A} 4$ (tableau III). Cholette et Mc Kellar (1990) ont montré qu'à $\mathrm{pH} 5,2$ et en présence de $2 \%$ de sel, l'activité aminopeptidasique est inférieure à $1 \%$ de son activité maximale ; l'affinité enzyme-substrat semble diminuer en même temps que le $\mathrm{pH}$. De même, Frey et al (1986) indiquent que la combinaison d'un $\mathrm{pH}$ bas $(\mathrm{pH} 5,0)$ et d'un taux élevé de sel $(5 \%)$ diminue considérablement l'activité proline et leucine aminopeptidasique de $L$ helveticus. II semble donc difficile de prévoir à partir de simples tests de laboratoire, I'intensité de proteolyse des fromages.

\section{Analyses sensorielles}

\section{Profil sensoriel des fromages}

Pour l'ensemble de l'expérimentation, les 48 fromages ont obtenu des notes moyennes de 6,50 et 6,02, respectivement pour la pâte et le goût. La note de typicité a atteint la valeur de 4,78 indiquant des fromages peu typés, et celle de l'amertume, 3,30 . Le caractère piquant est resté faible, avec des valeurs moyennes de 2,01 (tableau III).

\section{Influence des facteurs}

L'analyse de variance (tableau IV) montre que le facteur souche n'est pas significatif au seuil de $5 \%$ pour la plupart des variables ; par contre le facteur fromagerie intervient au niveau de plusieurs caractéristiques sensorielles ; il explique entre $28 \%$ et $60 \%$ des variations observées pour les caractéristiques de pâte (résistance, onctuosité, élasticité et hétérogénéité) ainsi que $32 \%$ des variations au niveau du critère acidité. Les fromages les plus onctueux $(6,37)$ sont issus de la fromagerie F5 ; ils apparaissent peu résistants $(4,78)$. Ces fromages présentent également à $20 \mathrm{~h}$ le rapport G/S le plus élevé $(50,28 \%)$. Les fromages les moins élastiques $(4,82$ et $4,55)$ sont fabriqués respectivement par les fromageries F4 et F2. Ils présentent également à 5 mois un rapport NPT/NS élevé. La texture du fromage semble donc liée à la composition physico-chimique du fromage à $20 \mathrm{~h}$ et aux phénomènes biochimiques qui se déroulent pendant l'affinage ; ces observations vont dans le sens de celles développées par Grappin et al (1993). Le facteur fromagerie qui regroupe la nature du lait, la technologie et les conditions d'affinage n'est donc pas sans importance dans la variation des critères sensoriels de pâte du fromage.

La répétabilité des analyses n'étant pas la même pour chacune des associations, aucune conclusion significative ne découle des notes d'amertume. Cependant, les valeurs données par chacun des mélanges montrent que les fromages fabriqués avec des souches de $L$ helveticus ont tendance 
à être plus amers $(A 1=3,95 ; A 2=3,86)$ que ceux fabriqués avec $L$ delbrueckii subsp lactis ( $A 3=2,69 ; A 4=2,71)$.

\section{Relations entre les caractéristiques sensorielles et les analyses physico-chimiques des fromages}

La fraction NPT/NT est corrélée positivement à l'intensité de goût $(r=0,527)$; cette observation a déjà été faite lors d'études précédentes sur le Cheddar (Aston et al, 1983) et sur le comté (Grappin et Berdagué, 1989). L'amertume est par contre corrélée négativement avec le rapport NPT/ NS $(r=-0,665)$, montrant que le critère sensoriel amer évolue dans le même sens que la fraction NS et en sens opposé à la fraction NPT. Berdagué et Grappin (1988) avaient déjà mis en évidence une corrélation positive entre l'amertume et le $\log$ (NS) d'une part $(r=0,72)$ et la somme des fractions caséines $(\gamma 2+\gamma$ en \%) d'autre part $(r=0,66)$. Dans notre étude, aucune relation significative n'a cependant été trouvée entre les fractions caséines et le critère d'amertume. Ardö et Mansson (1990) ont également montré que l'amertume diminue lorsque le taux d'acides aminés libres augmente.

Une étude des profils CLHP a été réalisée afin de mettre en évidence d'éventuelles relations entre la protéolyse secondaire des fromages et leurs caractéristiques sensorielles (amertume, typicité). Les fromages présentant toujours les mêmes pics majeurs (pics 1 à 4 , fig 2), le profil chromatographique a été découpé en 4 zones excluant ces pics (zone 1 : espace compris entre les pics 1 et 2 , zone 2 : espace compris entre les pics 2 et 3 , zone 3 : espace compris entre les pics 3 et 4 , zone 4 : espace restant). La surface cumulée des pics contenus dans chaque zone a été calculée, ainsi que le pourcentage par rapport à la surface totale. La matrice des corrélations n'a pas montré de corrélation significative entre la note de typicité et les différentes zones du profil CLHP ; par contre, la zone 4 est apparue corrélée positivement à la note d'amertume (fig 3 ). Dans la chromatographie en phase inverse (RP-CLHP), la phase stationnaire est apolaire; les composés apolaires sont retenus préférentiellement sur cette phase par des interactions de type hydrophobe. Les peptides amers, renfermant une majorité d'acides aminés hydrophobes (Lemieux et Simard, 1991 et 1992) devraient donc être davantage retenus sur la colonne que les peptides de nature hydrophile et donc sortir en fin de chromatogramme. Cela peut expliquer la corrélation positive observée entre l'amertume des fromages et la zone 4 du profil chromatographique. En étudiant la séparation de peptides amers isolés de Cheddar, Champion et Stanley (1982) avaient également observé que les fractions amères étaient plus hydrophobes

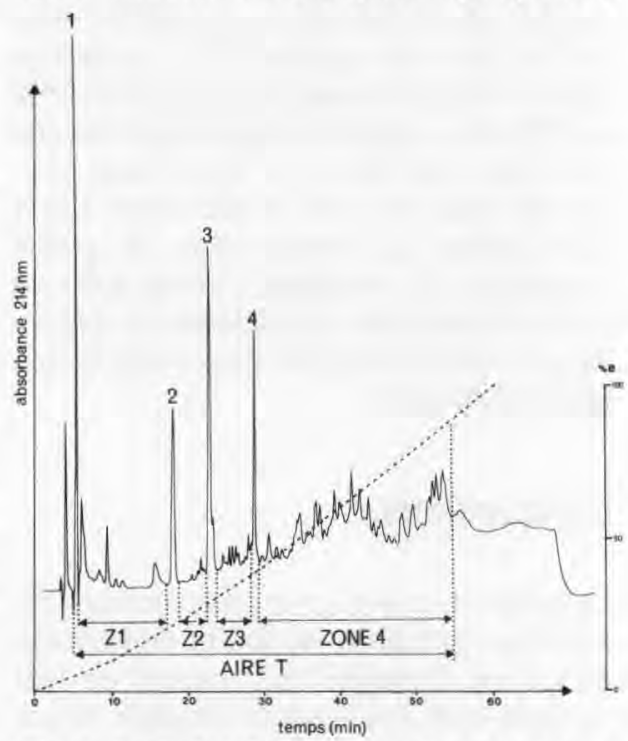

Fig 2. Profil CLHP de la fraction soluble dans l'eau d'un Comté.

HPLC profile of the water-soluble fraction of Comté. 


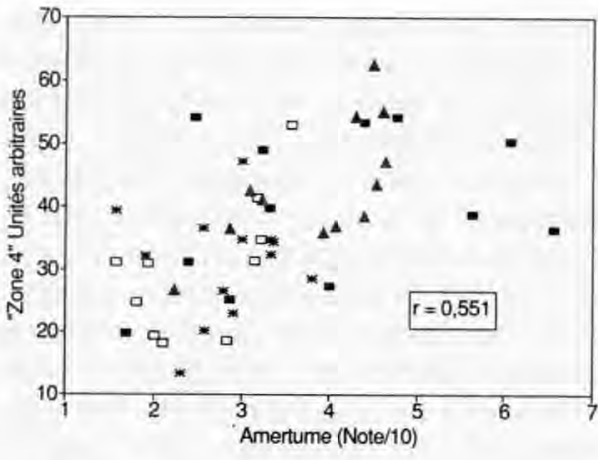

Fig 3. Relation entre la zone 4 du chromatogramme et la note d'amertume.

Relationship between zone 4 of the chromatogram and the bitterness score.

que les fractions non amères et qu'elles étaient plus riches en valine et leucine.

Une analyse de variance des différents paramètres calculés à partir des profils CLHP des 48 fromages (tableau IV) révèle que le facteur souche, significatif au seuil de $5 \%$ pour les variables pic 1 , zone 3 et zone 4 explique respectivement 19\%, 20\% et $22 \%$ des variations observées pour ces variables. Les pics 1, 2, 3 et 4 sont plus grands avec $A 3$ et $A 4$ et les zones 3 et 4 plus petites. La comparaison de profils peptidiques de fromages permet ainsi de mettre en évidence des différences de protéolyse liées à l'activité protéolytique des levains lactiques.

\section{CONCLUSIONS}

La mise en œuvre d'un petit nombre de souches isolées de levains et de fromage de Comté témoigne tout d'abord de leur capacité technologique en situation industrielle puisque les fromages fabriqués correspondaient tous à la norme de l'appellation. Nous avons pu confirmer l'influence du facteur souche sur la formation de pe- tits peptides et d'acides aminés dans le fromage au cours de l'affinage. Cet effet prépondérant de l'activité protéolytique des levains a déjà été montré lors d'une étude précédente (Delacroix-Buchet, 1986). Sur l'ensemble des fromages, parmi les souches testées, il ressort que les associations de $L$ delbrueckii subsp lactis (L85 et L90), produisent davantage d'acides aminés et de petits peptides que les autres associations. de $L$ helveticus. Cet aspect semble important à retenir puisque le critère sensoriel "intensité du goût» (indépendamment de sa finesse) est apparu corrélé à la fraction NPT/NT. Les souches destinées à la fabrication de Comté devront présenter un potentiel exopeptidasique suffisant afin de contribuer à la production d'acides aminés et de petits peptides dans le fromage, nécessaire à l'intensité du goût. Farkye et al (1990) ont suggéré que l'action peptidasique des levains est plus importante pour le développement de la flaveur du Cheddar, que l'activité protéinasique. La teneur en acides aminés et petits peptides dans le fromage ne doit cependant pas être excessive pour ne pas occasionner des défauts de lainure (Grappin et Berdagué, 1989). Par ailleurs, le facteur fromagerie (nature du lait, technologie, affinage) influence également la protéolyse du fromage; même si nous n'avons pu mettre en évidence de corrélation entre les teneurs en plasmine et plasminogène des laits utilisés et celle des caséines $\gamma$ et $\beta$ deg, nous savons que d'autres facteurs tels que la diffusion du sel ou bien la température d'affinage influencent la production d'acides aminés dans le fromage (Youssef, 1992).

Comme dans l'étude réalisée sur minifromages, on peut noter également qu'il est difficile de prévoir à partir des tests de laboratoire $\left(-\mathrm{NH}_{2}\right.$ et Lap), l'intensité de la protéolyse au niveau du fromage. Différentes analyses permettent d'apprécier la protéolyse du fromage. La mesure des 
groupements aminés libres par le réactif TNBS est bien corrélée à celle de l'azote soluble dans l'acide phosphotungstique dosé par la méthode de Kjeldahl ( $r=$ $0,951, N=48$ ) et peut donc remplacer avantageusement (dosage moins long) cette dernière méthode pour une estimation rapide de la protéolyse dans le fromage. La mesure de l'activité leucineaminopeptidasique à $20 \mathrm{~h}$ peut être maintenue comme témoin d'une activité peptidasique au sein du fromage mais ne nous permet pas d'envisager la protéolyse du fromage affiné. Le dosage des fractions caséines ne constituant pas un élément de distinction entre les souches de lactobacilles, il n'apparaît pas nécessaire de retenir l'électrophorèse comme technique d'appréciation de la protéolyse, liée à l'activité des ferments lactiques. Par contre, les profils CLHP devraient permettre d'apprécier plus finement la protéolyse sur fromages. Les premiers résultats obtenus lors de ce travail sont encourageants, puisque nous avons pu mettre en évidence des différences de profils peptidiques, les fromages amers présentant en fin de chromatogramme, une surface cumulée des pics plus importante que les fromages non amers. Cliffe et Law (1991) en travaillant sur l'accélération de l'affinage du Cheddar, ont détecté par CLHP, un pic dont la surface est bien corrélée à l'intensité de la flaveur. Par ailleurs, des travaux récents (Roudot-Algaron, 1992) ont permis d'identifier 35 composés contenus dans la fraction hydrosoluble. Ces composés peuvent se classer en 5 familles structurales : acides aminés $\mathrm{N}$-acylés, dipeptides linéaires, dipeptides cycliques, phosphopeptides et autres, ne provenant pas de la dégradation des protéines (acide citrique, 4-méthyl-5thiazoéthanol). Ces résultats nous permettent de penser qu'une meilleure connaissance de la fraction hydrosoluble devrait nous aider à mieux comprendre son rôle dans l'intensité de la flaveur.

\section{REMERCIEMENTS}

Nous remercions $S$ Pochet, pour la mise au point et la réalisation des dosages chromatographiques, E Aliphat, pour sa contribution aux analyses physico-chimiques, les fromageries et affineurs qui ont participé aux différentes expérimentations ainsi que tous les membres du jury d'analyse sensorielle.

Ce programme a reçu le soutien financier du Comité interprofessionnel du gruyère de Comté, de l'Association pour le développement de la recherche dans l'Industrie laitière et de l'Association nationale pour la recherche et la technologie (Bourse CIFRE de $Y$ Bouton, convention $\left.n^{\circ} 12 / 89\right)$.

\section{RÉFÉRENCES}

Anonyme (1982) Fromages et fromages fondus. Détermination de l'extrait sec total; méthode de référence. Norme FIL 4A, Bruxelles

Anonyme (1988) Fromages et fromages fondus. Détermination de la teneur en chlorures ; méthode par titrage potentiométrique. Norme FIL 88A, Bruxelles

Ardö Y, Mansson HL (1990) Heat treated Lactobacilli develop desirable aroma in low fat cheese. Scand Dairy Inf 4, 38-40

Aston JW, Dulley JR (1982) Cheddar cheese flavor. Aust J Dairy Technol 37, 59-64

Aston JW, Creamer LK (1986) Contribution of the components of the water-soluble fraction to the flavour of Cheddar cheese. N Z J Dairy Sci Technol 21, 229-248

Aston JW, Durward IG, Dulley JR (1983) Proteolysis and flavour development in Cheddar cheese. Aust J Dairy Technol 38, 55-59

Berdagué JL, Grappin R (1988) Affinage et qualité du gruyère de Comté. VI. Caractéristiques sensorielles des fromages. Lait 68 , 189-204

Berdagué JL, Jeunet R, Grappin R (1987) Affinage et qualité du gruyère de Comté. III. Fermentation lactique et teneur en acides gras volatils des fromages de Comté. Lait 67, 249-263

Biede SL, Hammond EG (1979) Swiss cheese flavor : II Organoleptic analysis. J Dairy Sci $62,238-248$ 
Bouton Y, Guyot P, Dasen A, Grappin R (1993) Activité protéolytique de souches de lactobacilles thermophiles isolées de levains et de comté. I. Validation sur minifromages des techniques de laboratoire. Lait 73, 265-279

Champion HM, Stanley DW (1982) HPLC separation of bitter peptides from Cheddar cheese. Can Inst Food Sci Technol J 15, 283-288

Cholette H, Mc Kellar RC (1990). Influence of $\mathrm{pH}$ on the properties of $L$ helveticus aminopeptidase. J Dairy Sci 73, 2278-2286

Cliffe AJ, Law BA (1991) A time course study of peptide production in accelerated-ripened Cheddar cheese using reverse phase high performance liquid chromatography. Food Biotechnol 5, 1-17

Collin JC, Berdagué JL, Dognin-Bergeret $M$, Grappin R (1987) Affinage et qualité du Gruyère de Comté. IV. Étude de la protéolyse. Lait 67, 299-318

Delacroix-Buchet A (1986) Les ferments lactiques thermophiles, étude de leur activité protéolytique. Rapport final, contrat CEE/ITG/ INRA N ${ }^{\circ} 1120 / 86$, code INRA9182A

Delacroix-Buchet A, Fournier S (1992) Protéolyse et texture des fromages à pâte cuite pressée. II. Influence de la chymosine et des conditions de fabrication. Lait 72, 53-72

Farkye NY, Fox PF, Fitzgerald GF, Daly C (1990) Proteolysis and flavour development in Cheddar cheese made exclusively with single strain proteinase-negative starters. $J$ Dairy Sci 73, 874-880

Frey JP, Marth EH, Johnson ME, Olson NF (1986) Heat and freeze-shocking cause changes in peptidase and protease activity of $L$ helveticus. Milchwissenschaft 41, 681-685

Grappin R, Berdagué JL (1989) Affinage et qualité du gruyère de Comté. VIII. Synthèse et conclusions. Lait 69, 183-196

Grappin R, Lefier D, Dasen A, Pochet S (1993) Characterizing ripening of Gruyère de
Comté : influence of time $x$ temperature and salting conditions on eye and slit formation. Int Dairy J 3, 313-328

Gripon JC, Desmazeaud MJ, Le Bars D, Bergère JL, (1975) Étude du rôle des microorganismes et des enzymes au cours de la maturation des fromages. II. Influence de la présure commerciale. Lait 55, 502-516

Heiss (1961) Essais de dosage de la matière grasse dans le fromage par des méthodes rapides. Dtsch Molk Ztg 82, 3, 67-70

Khalid NM, Marth EH (1990) Lactobacilli. Their enzymes and role in ripening and spoilage of cheese : a review. J Dairy Sci 73, 2669-2684

Kuchroo CN, Fox PF (1982) Soluble nitrogen in Cheddar cheese : Comparison of extraction procedures. Milchwissenschaft 37, 331-335

Lavanchy $P$, Bérodier $F$, Zannoni $M$, Noël $Y$, Adamo C, Squella J, Herrero L (1993) L'évaluation sensorielle de la texture des fromages à pâte dure ou semi-dure. Etude interlaboratoires. Lebensm Wiss Technol 26, 59-68

Lemieux L, Simard RE (1991) Bitter flavour in dairy products. I. A review of the factors likely to influence its development, mainly in cheese manufacture. Lait 71, 599-636

Lemieux L, Simard RE (1992) Bitter flavour in dairy products. II. A review of bitter peptides from caseins : their formation, isolation and identification, structure masking and inhibition. Lait 72, 335-382

Roudot-Algaron F (1992) Influence de la fraction hydrosoluble sur la flaveur du fromage. Identification organoleptique des principaux constituants. Thèse de doctorat, Paris VII, France

Uriel J (1966) Méthode d'électrophorèse des gels d'acrylamide-agarose. Bull Soc Chim Biol 48, 962-982

Youssef YB (1992) Factors affecting proteolytic action of Lactococcus lactis in cheese. Ph D thesis, Agricultural university, Wageningen 